\title{
Recent infection testing algorithm (RITA) applied to new HIV diagnoses in England, Wales and Northern Ireland, 2009 to 2011
}

\author{
A Aghaizu (adamma.aghaizu@phe.gov.uk) ${ }^{1}$, G Murphy², J Tosswill ${ }^{2}$, D DeAngelis ${ }^{3}$, A Charlett ${ }^{1}, 0$ N Gill ${ }^{1}$, H Ward ${ }^{4}$, S Lattimore ${ }^{1}$, R \\ D Simmons ${ }^{5}$, V Delpech ${ }^{1}$ \\ 1. HIV and STI Department, Centre for Infectious Disease Surveillance and Control, Public Health England, Colindale, London, \\ United Kingdom \\ 2. Virus Reference Department, Microbiology Services Colindale, London, United Kingdom \\ 3. MRC Biostatistics Unit, Institute of Public Health, Cambridge, United Kingdom \\ 4. Department of Infectious Disease Epidemiology, Imperial College London, United Kingdom \\ 5. MRC Clinical Trials Unit, London, United Kingdom
}

Citation style for this article:

Aghaizu A, Murphy G, Tosswill J, DeAngelis D, Charlett A, Gill ON, Ward H, Lattimore S, Simmons RD, Delpech V. Recent infection testing algorithm (RITA) applied to new HIV diagnoses in England, Wales and Northern Ireland, 2009 to 2011. Euro Surveill, 2014:19(2):pii=20673. Available online: http://www.eurosurveillance.org/ ViewArticle.aspx?Articleld $=20673$

In 2009, Public Health England (PHE) introduced the routine application of a recent infection testing algorithm (RITA) to new HIV diagnoses, where a positive RITA result indicates likely acquisition of infection in the previous six months. Laboratories submit serum specimens to PHE for testing using the HIV $1 / 2$ gO AxSYM assay modified for the determination of HIV antibody avidity. Results are classified according to avidity index and data on $\mathrm{CD}_{4}$ count, antiretroviral treatment and the presence of an AIDS-defining illness. Between 2009 and 2011, 38.4\% (6,966/18,134) of new HIV diagnoses in England, Wales and Northern Ireland were tested. Demographic characteristics of those tested were similar to all persons with diagnosed HIV. Overall, recent infection was $14.7 \%(1,022 / 6,966)$ and higher among men who have sex with men (MSM) $(22.3 \%, 720 / 3,223)$ compared with heterosexual men and women $(7.8 \%, 247 / 3,164)$. Higher proportions were among persons aged 15-24 years compared with those $\geq 50$ years (MSM 31.2\% (139/445) vs 13.6\% (42/308); heterosexual men and women $17.3 \%(43 / 249)$ vs $6.2 \%$ (31/501)). Among heterosexual men and women, black Africans were least likely to have recent infection compared with whites (4.8\%, 90/1,892 vs $13.3 \%$, 97/728; adjusted odds ratio: $0.6 ; 95 \% \mathrm{Cl}: 0.4-0.9)$. Our results indicate evidence of ongoing HIV transmission during the study period, particularly among MSM.

\section{Introduction}

With over 6,000 new human immunodeficiency virus (HIV) diagnoses in 2011 in the United Kingdom (UK) [1] and a steady increase in the number and proportion of new diagnoses among men who have sex with men (MSM), as well as an increase among UK-acquired infections among heterosexual men and women [2], controlling the HIV epidemic continues to be a public health priority. To ensure public health interventions are implemented efficiently and effectively, an accurate, regular assessment of the epidemic is needed.

HIV incidence, the rate of new infections, is considered to be the most valuable measure for describing the current dynamics of the epidemic. Determining the rate of new infections remains challenging as there is a prolonged asymptomatic period and therefore, in the absence of screening, diagnosis can be delayed for several years. One approach is to use positivity for biomarkers to distinguish recently acquired from longstanding HIV infections from a single sample [3]. Some institutions have incorporated biomarker-based assays as part of the routine surveillance of HIV, such as the Institut de Veille Sanitaire in France [4], and the Centers for Disease Control and Prevention in the United States $[5,6]$. A technical guide on how to implement testing for recent infection has been developed by the European Centre for Disease Prevention and Control [7].

In 1998, Public Health England (PHE), formerly the Health Protection Agency, introduced the use of a biomarker for the estimation of recent HIV infection among MSM attending sentinel sexual health clinics. This technology has since been applied to distinct HIV incidence research studies and sentinel surveillance sites [8,9]. In 2009, a biomarker testing programme was rolled out in England, Wales and Northern Ireland, offering testing to individuals newly diagnosed with HIV [10]. In the UK, the epidemic is concentrated in two key risk populations: (i) MSM who are mostly white and acquired HIV in the UK; and (ii) heterosexual men and women of black African ethnicity, of whom a large proportion acquired HIV abroad.

In this article, we review the implementation of the first three years of the programme and examine factors 
associated with biomarker test results indicative of recent infection among persons newly diagnosed with HIV infection.

\section{Methods}

\section{Surveillance of recently}

\section{acquired HIV infections}

PHE collates national data on all new diagnoses of HIV, AIDS and deaths among people living with HIV along with demographic and epidemiological information for individuals aged over 15 years. Since 2009, laboratories in England, Wales and Northern Ireland have been sending specimens from persons newly diagnosed with HIV to the Virus Reference Department at PHE Colindale for testing using a recent infection testing algorithm (RITA) to identify HIV infections archetypal of a recent infection. Results are linked to the new HIV diagnoses database using pseudo-anonymised data on the diagnosis site, soundex (scrambled surname code) [11], date of birth and sex. Samples taken from the patient more than four months after the initial diagnosis are excluded from analyses due to the reduced likelihood of these being a recent infection.

The RITA classifies new diagnoses with an avidity index $<80 \%$ as positive (a likely recent infection) unless other available clinical information, which completes the algorithm, indicates a likely long-standing infection, i.e. a CD4 count $<200$ cells $/ \mathrm{mm}^{3}$ at diagnosis, a report of an AIDS-defining illness within a year of diagnosis or history of antiretroviral treatment. A RITA-positive result is indicative of likely acquisition of infection around six months before diagnosis. In this paper, we refer to RITA-positive diagnoses as 'recent infections'. The avidity assay results are returned to the clinician via local laboratories; at patient level, clinicians interpret the avidity results alongside other test results and in context of information in case notes.

\section{Laboratory testing}

Testing is carried out using the AxSYM assay HIV $1 / 2$ g0 (Abbott, United States) modified to determine antibody avidity, as described elsewhere [12]. This assay indirectly measures the HIV antibody-antigen bond strength or 'avidity', which is typically weaker during the initial stages of the infection [13]. Test results are reported as an index, with $80 \%$ used as a positive cutoff value; results between $75 \%$ and $85 \%$ are retested and the mean of the two results is used.

\section{Statistical analysis}

Data management and analyses were performed using Microsoft Access 2007 and STATA 12.0 (Stata Statistical Software: Release 12, United States). To examine characteristics of individuals with recent infection, we stratified by exposure group (MSM, heterosexual men and women and other) and performed single- and multivariable analyses using logistic regression including any variables in the final model where a hypothesis test on the regression parameters resulted in p<o.2.

\section{Results}

Testing coverage and representativeness

Between 2009 and 2011, there were a total of 18,134 new HIV diagnoses in England, Wales and Northern Ireland. Over this period, 10,088 samples were received for avidity testing, of which 6,966 (69\%) were linked to a new diagnosis report and taken within four months of the diagnosis date. Avidity testing coverage was therefore $38 \%$ for the new 18,134 diagnoses over the three-year period as a whole, increasing from $24 \%$ $(1,479 / 6,234)$, from 41 laboratories, in 2009 to $52 \%$ $(3,069 / 5,894)$, from 83 laboratories, in 2011. Coverage was broadly similar across subpopulations apart from slightly more testing among individuals from London and individuals of black Caribbean and other black ethnicity, and less testing among people who inject drugs (PWID); however, numbers were small among PWID (Table 1). The mean age of individuals tested for recent infection was 35.6 years (standard deviation (SD): 10.5) for MSM, 36.6 years (SD: 10.5) for heterosexual women and 41.3 years (SD: 10.5$)$ for heterosexual men, similar to all individuals newly diagnosed in these risk groups: 36.2 years (SD: 10.7$)$ among MSM, 36.4 years (SD: 10.1) among heterosexual women and 41.2 years (SD: 10.9 ) among heterosexual men.

\section{Recent infections among new HIV diagnoses} After reclassifying individuals whose samples had an avidity score $<80 \%$ and a CD4 count $<200$ cells/ $\mathrm{mm}^{3}(\mathrm{n}=61)$, diagnosis of an AIDS-defining illness $(n=5)$ or antiretroviral treatment before or at the time the sample was taken (for example, pre- or post-exposure prophylaxis) $(n=44)$ as having longstanding infections, the overall proportion of recent infection was $14.7 \%(1,022 / 6,966)$ (Figure 1). The highest proportion of recent infection was among MSM, $22.3 \%(720 / 3,223)$ compared with $7.8 \%$ $(247 / 3,164)$ among heterosexual men and women, 5.6\% (6/108) among PWID and 10.4\% (49/471) among 'other'. The proportion was slightly higher among heterosexual women $(8.1 \%, 153 / 1,892)$ compared with heterosexual men $(7.4 \%, 94 / 1,272)$ and the proportions were similar across the categories for all three years (data not shown).

Among MSM, higher proportions of recent infections were observed among younger individuals, with the highest among those aged 15-24 years compared with those aged 50 years and over (31.2\%, 139/445 vs $13.6 \%, 42 / 308$ ) (Table 2). Among MSM, the proportions of recent infections were similar across ethnicities, apart from among black African MSM where it was lower $(13.9 \%(10 / 72)$ compared with $22.3 \%(575 / 2,584)$ among those who were white. The proportions of recent infections were similar among MSM born in the UK and abroad; however, it was slightly lower among MSM reported as having acquired their infection abroad than among those reported as having acquired their infection in the UK (17.4\%, $179 / 1,027$ vs $24.6 \%, 541 / 2,196)$. Multivariable analyses showed younger age (15-24 
TABLE 1

Proportion of new HIV diagnoses tested for recent infection in England, Wales and Northern Ireland, 2009-2011

\begin{tabular}{|c|c|c|c|}
\hline \multirow{2}{*}{ Characteristic } & \multicolumn{3}{|c|}{$\%$ coverage ( $\mathrm{n}$ tested/N diagnosed) } \\
\hline & 2009 & 2010 & 2011 \\
\hline Total & $23.7(1,479 / 6,234)$ & $40.3(2,418 / 6,006)$ & $52.0(3,069 / 5,894)$ \\
\hline \multicolumn{4}{|l|}{ Transmission route } \\
\hline Men who have sex with men & $26.3(656 / 2,496)$ & $41.6(1,063 / 2,558)$ & $57.5(1,504 / 2,617)$ \\
\hline Heterosexual men & $21.8(272 / 1,248)$ & $38.3(447 / 1,166)$ & $48.1(553 / 1,149)$ \\
\hline Heterosexual women & $23.1(434 / 1,878)$ & $41.2(692 / 1,678)$ & $51.7(766 / 1,481)$ \\
\hline People who inject drugs & $15.2(20 / 132)$ & $35.9(46 / 128)$ & $36.8(42 / 114)$ \\
\hline Other & $20.2(97 / 480)$ & $35.7(170 / 476)$ & $38.3(204 / 533)$ \\
\hline \multicolumn{4}{|l|}{ Age group in years } \\
\hline $15-24$ & $24.7(163 / 661)$ & $40.9(259 / 633)$ & $56.4(345 / 612)$ \\
\hline $25-34$ & $23.8(502 / 2,106)$ & $40.6(797 / 1,964)$ & $54.7(1,070 / 1,956)$ \\
\hline $35-49$ & $24.0(638 / 2,660)$ & $39.8(1,033 / 2,594)$ & $50.3(1,258 / 2,499)$ \\
\hline$\geq 50$ & $21.8(176 / 807)$ & $40.4(329 / 815)$ & $47.9(396 / 827)$ \\
\hline \multicolumn{4}{|l|}{ Ethnicity } \\
\hline White & $22.5(693 / 3,076)$ & $39 \cdot 3(1,148 / 2,917)$ & $53.9(1,670 / 3,100)$ \\
\hline Black African & $23.1(480 / 2,082)$ & $40.5(747 / 1,845)$ & $49.8(826 / 1,659)$ \\
\hline Black Caribbean & $31.6(75 / 237)$ & $52.0(102 / 196)$ & $59.6(99 / 166)$ \\
\hline Black other & $31.3(40 / 128)$ & $50.0(64 / 128)$ & $64.2(61 / 95)$ \\
\hline Indian/Pakistani/Bangladeshi & $26.2(28 / 107)$ & $34.1(46 / 135)$ & $45.6(52 / 114)$ \\
\hline Other & $27.0(163 / 604)$ & $39.6(311 / 785)$ & $47.5(361 / 760)$ \\
\hline \multicolumn{4}{|l|}{ Country of birth } \\
\hline United Kingdom & $20.7(460 / 2,218)$ & $42.1(879 / 2,087)$ & $56.3(1,128 / 2,004)$ \\
\hline Abroad & $25.4(1,019 / 4,016)$ & $39.4(1,545 / 3,919)$ & $49.9(1,941 / 3,890)$ \\
\hline \multicolumn{4}{|l|}{ Probable country of infection } \\
\hline United Kingdom & $27.5(654 / 2,378)$ & $44 \cdot 5(1,073 / 2,411)$ & $59.4(1,425 / 2,397)$ \\
\hline Abroad & $21.4(825 / 3,856)$ & $37.4(1,345 / 3,595)$ & $47.0(1,644 / 3,497)$ \\
\hline \multicolumn{4}{|l|}{ Region of diagnosis } \\
\hline London & $33.5(937 / 2,801)$ & $44.8(1,217 / 2,714)$ & $59.8(1,559 / 2,607)$ \\
\hline
\end{tabular}

HIV: human immunodeficiency virus.

years) (adjusted odds ratio (AOR): $1.8 ; 95 \% \mathrm{Cl}: 1.2-2.8$ and 25-34 years AOR: $1.6 ; 95 \% \mathrm{Cl}: 1.1-2.3)$ and the UK as the probable country of infection (AOR: $1.5 ; 95 \% \mathrm{Cl}$ : 1.2-1.8) were associated with a likely recent infection.

Among heterosexual men and women, the highest proportions of recent infection were among 15-24 year-old women $(19.5 \%, 38 / 195)$ and $25-34$ year-old men $(6.4 \%, 15 / 234)$. Lower proportions were observed among persons born abroad (6.4\%, 163/2,554 vs $13.8 \%, 84 / 610)$ and those reported to have acquired their infection abroad compared with in the UK $5.5 \%$, $126 / 2,302$ vs $14.0 \%, 121 / 862$ ). Of the four heterosexual men and women of Chinese ethnicity tested for recent infection, none were recently infected and only one among the Indian/Pakistani/Bangladeshi group $(n=46)$, but it should be noted that the numbers were small. Black African heterosexual men and women had a considerably lower proportion of recent infections $(4.8 \%, 90 / 1,892)$ compared with those who were white $(13.3 \%, 97 / 728)$; individuals in the 'black other' group had the highest proportion (14.8\%, 12/81). Multivariable analyses showed ethnicity and country of infection to be associated with a recent infection: black Africans were less likely (AOR: 0.6; 95\% CI: 0.4-0.9), whereas those of 'black other' ethnicity (AOR: 2.4; 95\% Cl: 1.1-5.3) and those with the UK as the probable country of infection (AOR: $1.7 ; 95 \% \mathrm{Cl}: 1.3-2.4$ ) were the most likely to be recently infected.

\section{Relationship between CD4 count}

and recent infection status

There was a strong association and a significant positive trend between $\mathrm{CD}_{4}$ counts $>200$ cells $/ \mathrm{mm}^{3}$ and recent infection classifications. Among MSM, only $11.4 \%(68 / 595)$ of individuals with a CD4 count between 
Flowchart of samples included in analyses and categorised according to the recent infection testing algorithm (RITA), England, Wales and Northern Ireland, 2009-2011

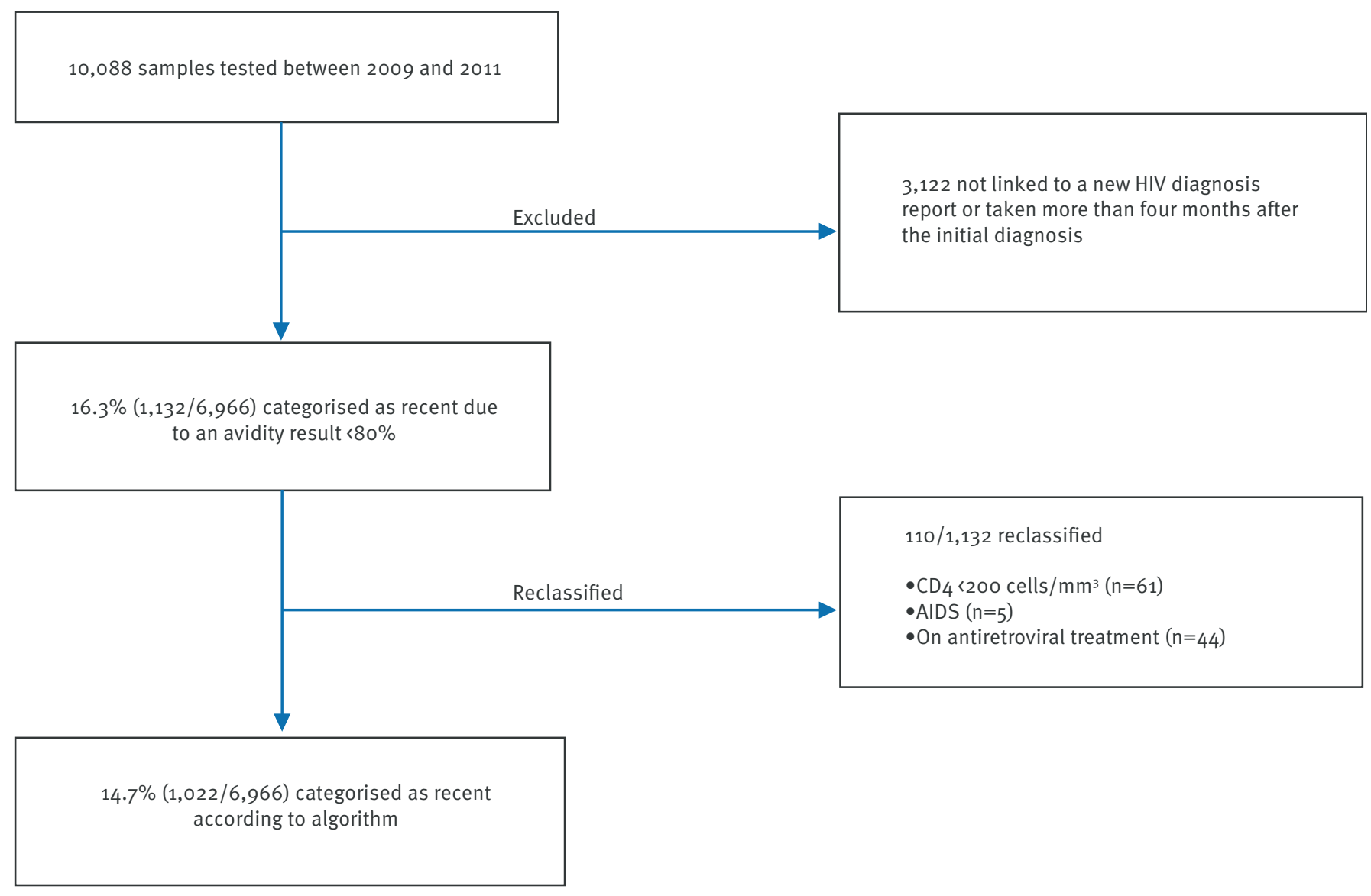

AIDS: acquired immunodeficiency syndrome; HIV: human immunodeficiency virus.

$>200$ and $\leq 350$ cells $/ \mathrm{mm}^{3}\left(\leq 350\right.$ cells $/ \mathrm{mm}^{3}$ is the definition of a late diagnosis, at which point antiretroviral treatment should have started [14]), were classified as likely to have acquired their infection recently compared with $43.5 \%$ (37/85) with a CD4 count $>1,000$ cells $/ \mathrm{mm}^{3}$. Among heterosexual men and women, this was slightly lower, with the proportion of recent infection $5.8 \%$ (38/660) among those with a $\mathrm{CD}_{4}$ count between $>200$ and $\leq 350$ cells $/ \mathrm{mm}^{3}$ and $31.9 \%(23 / 72)$ among those with a CD4 count $>1,000$ cells $/ \mathrm{mm}^{3}$. A recent infection diagnosis was more likely if the individual had a CD4 count $>1,000$ cells $/ \mathrm{mm}^{3}$, compared with those with a CD4 count between $>200$ and $\leq 350$ cell/mm ${ }^{3}$ (AOR for MSM: 6.0, 95\% Cl: 3.7-9.9; AOR for heterosexual men and women: 7.6, 95\% Cl: 4.2-13.7).

\section{Discussion}

This study, covering the first three years of the implementation of a RITA to national surveillance of HIV diagnoses, indicates a high level of ongoing transmission among key populations in England, Wales and Northern Ireland during the study period. Our findings indicate that MSM remain the group at greatest risk of HIV infection, with one in five men diagnosed likely to have acquired their infection recently. As may be expected, younger age, high CD4 count and the UK being the probable country of infection were associated with likely recent acquisition of infection. Nevertheless, a substantial number of recent infections were seen also among MSM aged 50 years and over. Of note, there were no substantial differences by ethnicity or country of birth, indicating high levels of transmission regardless of these characteristics.

Among heterosexual men and women, the proportions of recent infection were lower than in MSM, particularly among those born abroad. Younger age, high CD4 count and the UK being the most probable country of infection were also associated with a likely recent infection in this group. There was considerable variation by ethnicity, with black Africans less than half as likely to have recently acquired infection at the time of diagnosis compared with those who were white. Interestingly, the 'black other' group, representing possibly those 


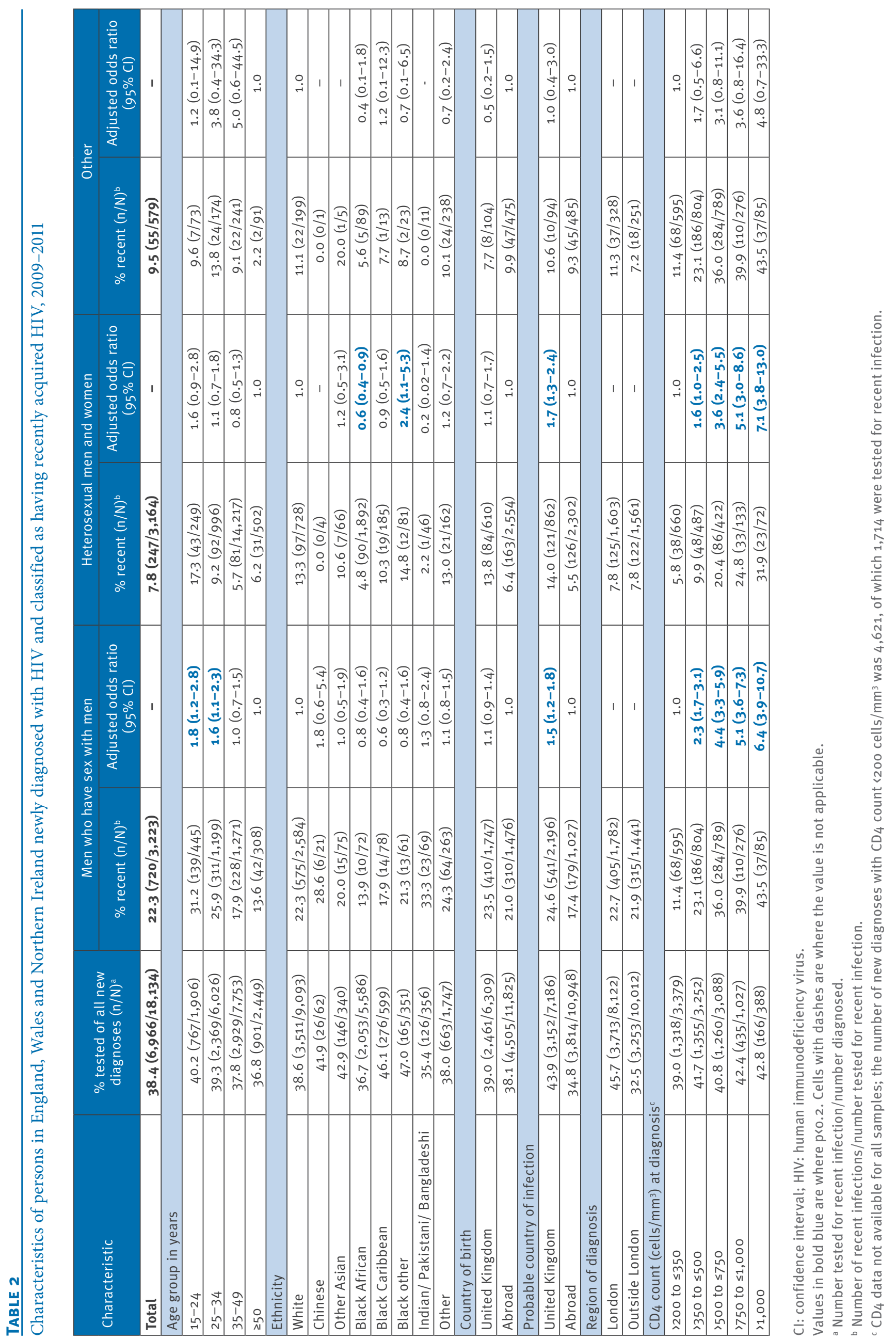


that identify as black British, had the highest odds of a likely recent infection at the time of diagnosis.

There are several limitations to our study. Firstly, the cut-off used for the avidity assay ( $80 \%)$ is based on a longitudinal seroconversion panel mean [15] with a duration of recency of six months for $58 \%$ of individuals and less than a year for $88 \%$ [16]. It is therefore likely that the proportions presented are an underestimate due to the limited sensitivity of the assay. Furthermore, the specificity of the test is not well understood, and thus the extent to which the algorithm may misclassify cases. In a separate study, we examined the number of recent infection classifications when applying the algorithm to 1,270 specimens from persons known to have been infected for more than a year. We found that the proportion misclassified, termed the false recent rate [17], was $1.3 \%(17 / 1,270)$. This implies that in the study presented here, up to $91(8.8 \%)$ of recent cases may have had an infection for more than a year, resulting in the overall proportion of recent infection $13.4 \%$ $(931 / 6,966)$. Also, it should be noted that CD4 information was not available for $10 \%(718 / 6,966)$ of cases, among whom the proportion of recent infections was $11.4 \%(82 / 718)$.

Secondly, HIV diagnoses are subject to testing patterns and therefore the absolute numbers and proportions need to be considered in the context of testing frequencies. Sexual health clinic data show MSM test more frequently than heterosexual men and women [1] and we undertook a recent study demonstrating regular testers are more likely to be diagnosed close to the time of infection [18]. Therefore, the higher proportions of recent infection among MSM will be partly attributable to the difference in testing patterns. Further study is needed to evaluate the extent to which lower proportions of recent infection among heterosexual men and women are due to infections acquired abroad or barriers to testing. Nevertheless, a substantial proportion of the recent infections in this group were reported to have been acquired in the UK, which is in line with findings of other studies $[2,10]$.

Thirdly, as coverage of testing for the three years combined was only $38 \%$, there is potential for selection bias. However, we found no major differences when we compared the demographic variables of those tested to all persons newly diagnosed (Table 1 ).

We found a positive association between recent infection and high $\mathrm{CD}_{4}$ count, both indicators of early-stage disease. Studies have shown that the mean CD4 count before seroconversion among MSM to be about 1,000 cells $/ \mathrm{mm}^{3}$, about 780 cells $/ \mathrm{mm}^{3}$ six months after infection and about 670 cells $/ \mathrm{mm}^{3}$ a year after infection, though with wide variations within and between individuals [19]. Among HIV-negative African populations, observations of median $\mathrm{CD}_{4}$ counts varied from 640 cells $/ \mathrm{mm}^{3}$ in Ethiopia [20] to 1,160 cells $/ \mathrm{mm}^{3}$ in Uganda [21,22]. Particularly among individuals with
HIV infection, it is not uncommon for $\mathrm{CD}_{4}$ counts to double or halve within eight weeks of an initial count, with an average variation of $25 \%$ from the mean over this period [23]. Therefore, there is considerable uncertainty in the expected $\mathrm{CD}_{4}$ counts within the first six months or year of infection, which may explain why the proportion of likely recent infection is not higher among those with $\mathrm{CD}_{4}$ counts similar to persons who are HIV negative.

It is known that $\mathrm{CD}_{4}$ counts can drop during seroconversion [24]; if below 200 cells $/ \mathrm{mm}^{3}$, according to the algorithm used in this study, individuals would be reclassified as having a long-standing infection $(n=61)$, potentially slightly underestimating the proportion of recent infection.

Along with France and the United States, the UK is one of the first countries to apply a RITA to routine case-based surveillance data. The UK uses the AxSYM assay modified for the determination of antibody avidity, whereas BED capture enzyme immunoassay (BED(EIA) is currently the assay of choice in the United States [5] and enzyme immunoassay for recent infection (EIA-RI) in France [4]. Each of these tests has a different mean duration of recency, making direct comparisons difficult. The coverage of testing was higher in France (77\% between 2003 and 2006) and lower in the United States (17\% in 2006) $[4,25]$. All three countries have found the highest proportions of likely recent infection among MSM. In France, this proportion was $43 \%$ among MSM, compared with $16 \%$ among heterosexual men and women and lower among those with sub-Saharan nationality compared with those who were French nationals ( $8 \%$ vs $34 \%$ ) $[4,25]$. In the United States, incidence estimates based on test for recent infection data showed that $53 \%$ of incident infections were among MSM and $45 \%$ among persons of black ethnicity [25].

In conclusion, routine surveillance of recent infection with HIV using a biomarker among those diagnosed is feasible in countries where case-based surveillance of HIV infection is in place. Our findings indicate that transmission is high and ongoing in England, Wales and Northern Ireland, and confirm that MSM are disproportionately affected by new infections. Such findings suggest prevention efforts to reduce HIV transmission among MSM should be aimed at all ages and ethnic backgrounds, irrespective of country of birth. Modelling studies illustrate interventions with the greatest impact need to target MSM with recent, undiagnosed infections $[26,27]$ and the RITA could be key in identifying persons in their networks through targeted partner notification. Further work is needed to evaluate RITA as a tool for accelerated partner notification. Better characterisation of HIV incidence assays is currently underway by the Consortium for the Evaluation and Performance of HIV Incidence Assays, a Bill and Melinda Gates-funded project [28]. 
Although the surveillance data in this study may not reflect HIV incidence in the population, they have been instrumental in demonstrating sustained high rates of recent transmission among persons diagnosed. The next steps are to convert these data into populationbased HIV incidence estimates. This will entail applying a sampling frame that accounts for the variation in testing patterns among subpopulations diagnosed and the probability that a person is diagnosed in the recent period of their infection $[25,29]$.

\section{Acknowledgments}

We gratefully acknowledge the continuing collaboration of clinicians, microbiologists, immunologists, public health practitioners, occupational health doctors and nurses and other colleagues who contribute to the surveillance of HIV and STIs in the UK, the HIV Incidence Advisory Group and Grace Mensah and Josephine Morris for performing the laboratory testing.

\section{Conflict of interest}

None declared.

\section{Authors' contributions}

All authors contributed to the design of the study. AA led on the data analysis and drafting of the manuscript supported by VD, GM, JT, DD, AC and HW. All authors commented on drafts of the manuscript and approved the final version.

\section{References}

1. Aghaizu A, Brown AE, Nardone A, Gill ON, Delpech VC, The HIV and AIDS Reporting Team, et al. HIV in the United Kingdom: 2013 report. Data to end December 2012. London: Public Health England; November 2013. Available from: http://www.hpa.org. uk/webc/HPAwebFile/HPAweb_C/1317140300680

2. Rice BD, Elford J, Yin Z, Delpech VC. A new method to assign country of HIV infection among heterosexuals born abroad and diagnosed with HIV. AIDS, 2012;26(15):1961-6. http://dx.doi.org/10.1097/QAD.0b013e3283578b80

3. Janssen RS, Satten GA, Stramer SL, Rawal BD, O'Brien TR, Weiblen B], et al. New testing strategy to detect early HIV-1 infection for use in incidence estimates and for clinical and prevention purposes. JAMA. 1998;280(1):42-8.

http://dx.doi.org/10.1001/jama.280.1.42

4. Semaille C, Cazein F, Pillonel J, Lot F, Le Vu S, Pinget R, et al. Four years of surveillance of recent HIV infections at country level, France, mid 2003-2006: experience and perspectives. Euro Surveill. 2008;13(36):pii=18968. Available from: http:// www.eurosurveillance.org/ViewArticle.aspx?Articleld $=18968$

5. Hall HI, Song R, Rhodes P, Prejean J, An Q, Lee LM, et al. Estimation of HIV incidence in the United States. JAMA. 2008;300(5):520-9.

http://dx.doi.org/10.1001/jama.300.5.520

6. Lee LM, McKenna MT. Monitoring the incidence of HIV Infection in the United States. Public Health Rep. 2007;122 Suppl 1:72-9.

7. European Centre for Disease Prevention and Control (ECDC). Monitoring recently acquired HIV infections in the European context. Stockholm: ECDC: 2013. Available from: http://www. ecdc.europa.eu/en/publications/Publications/monitoringrecently-acquired-HIV-infections-european-context.pdf

8. Fisher M, Pao D, Murphy G, Dean G, McElborough D, Homer G, et al. Serological testing algorithm shows rising HIV incidence in a UK cohort of men who have sex with men: 10 years application. AIDS. 2007; 21(17):2309-14 http://dx.doi.org/10.1097/QAD.0b013e3282efgfed

9. Sudarshi D, Pao D, Murphy G, Parry J, Dean G, Fisher M. Missed opportunities for diagnosing primary HIV infection. Sex Transm Infect. 2008;84(1):14-6.

http://dx.doi.org/10.1136/sti.2007.026963

10. Health Protection Agency (HPA). New HIV and AIDS diagnoses and deaths in the United Kingdom in 2011. News archive. Volume 6 No. 16; 20 April 2012. London: HPA; 2012. Available from: http://www.hpa.org.uk/hpr/archives/2012/news1612. htm\#idus

11. Mortimer JY, Salathiel JA. 'Soundex' codes of surnames provide confidentiality and accuracy in a national HIV database. Commun Dis Rep CDR Rev. 1995;5(12):R183-6.

12. Suligoi B, Galli C, Massi M, Di Sora F, Sciandra M, Pezzotti P, et al. Precision and accuracy of a procedure for detecting recent human immunodeficiency virus infections by calculating the humbibody avidity index by an automated immunoassay-based method. I Clin Microbiol. 2002;40(11):4015-20. method. J Clin Microbiol. 2002;40(11):4015-20.
http://dx.doi.org/10.1128/JCM.40.11.4015-4020.2002

13. Murphy G. Parry JV. Assays for the detection of recent infections with human immunodeficiency virus type 1. Euro Surveill. 2008;13(36):pii=18966. Available from: http://www. eurosurveillance.org/ViewArticle.aspx?Articleld $=18966$

14. Gazzard BG, Anderson J, Babiker A, Boffito M, Brook G, Brough $\mathrm{G}$, et al. British HIV Association guidelines for the treatment of HIV-1-infected adults with antiretroviral therapy 2008. HIV Med. 2008;9(8):563-608. http://dx.doi.org/10.1111/j.1468-1293.2008.00636.x

15. Suligoi B, Massi M, Galli C, Sciandra M, Di Sora F, Pezzotti P, et al. Identifying recent HIV infections using the avidity index and an automated enzyme immunoassay. I Acquir Immune Defic Syndr. 2003;32(4):424-8.

http://dx.doi.org/10.1097/00126334-200304010-00012

16. Sweeting MJ, De Angelis D, Parry J, Suligoi B. Estimating the distribution of the window period for recent HIV infections: a comparison of statistical methods. Stat Med. 2010;29(30):3194-202. http://dx.doi.org/10.1002/sim.3941

17. Murphy G, Aghaizu A, Tosswill J, Smith R, Mensah G, Delpech $\mathrm{V}$, et al. Recent Infection Test Algorithm (RITA): determining an assay False Recent Rate in a national survey of new HIV diagnoses. TUPE204- poster exhibition. XIX International AIDS Conference, 22-27 July 2012, Washington, DC, United States. Abstract available from: http://pag.aids2012.org/abstracts. aspx?aid $=11522$

18. Rayment M, Aghaizu A, Tosswill J, Murphy G, Hughes G, Delpech V. O1 14 Investigating the recent infection testing
algorithm (RITA): predictors of recent HIV infection among GUM clinic attendees. Sex Transm Infect. 2012; 88(A5 A6). Available from: http://sti.bmj.com/content/88/Suppl_1/A5.3.full. pdf+html

19. Stein DS, Korvick JA, Vermund SH. CD4+ lymphocyte cell enumeration for prediction of clinical course of human immunodeficiency virus disease: a review. J Infect Dis. 1992;165(2):352-63 http://dx.doil.org/10.1093/infdis/165.2.352

20. Kassu A, Tsegaye, Petros B, Wolday D, Hailu E, Tilahun T, et al. Distribution of lymphocyte subsets in healthy human immunodeficiency virus-negative adult Ethiopians from two geographic locales. Clin Diagn Lab Immunol. 2001;8(6):1171-6.

21. Tugume SB, Piwowar EM, Lutalo T, Mugyenyi PN, Grant RM, Mangeni FW, et al. Hematological reference ranges among healthy Ugandans. Clin Diagn Lab Immunol. 1995;2(2):233-5.

22. Williams BG, Korenromp EL, Gouws E, Schmid GP, Auvert $B$, Dye $C$. HIV infection, antiretroviral therapy, and $C D_{4}+$ cell count distributions in African populations. J Infect Dis. 2006;194(10):1451-8. http://dx.doi.org/10.1086/508206

23. Hughes MD, Stein DS, Gundacke HM, Valentine FT, Phair JP, Volberding PA. Within-subject variation in $C D_{4}$ lymphocyte count in asymptomatic human immunodeficiency virus infection: implications for patient monitoring. I Infect Dis. infection: implications 1994;169(1):28-36.

http://dx.doi.org/10.1093/infdis/169.1.28

24. Lodi S, Phillips A, Touloumi G, Geskus R, Meyer L, Thiebaut R, et al. Time from human immunodeficiency virus seroconversion to reaching $C D 4+$ cell count thresholds $<200,<350$, and $<500$ cells $/ \mathrm{mm}^{3}$ : assessment of need following changes in treatment guidelines. Clin Infect Dis. 2011;53(8):817-25. $\mathrm{http}: / / \mathrm{dx}$.doi.org/10.1093/cid/cir494

25. Karon JM, Song R, Brookmeyer R, Kaplan EH, Hall HI. Estimating HIV incidence in the United States from HIV/AIDS surveillance data and biomarker HIV test results. Stats Med. 2008;27(23):4617-33. http://dx.doi.org/10.1002/sim.3144

26. Marks G, Crepaz N, Janssen RS. Estimating sexual transmission of HIV from persons aware and unaware that they are infected with the virus in the USA. AIDS. 2006;20(10):1447-50. http://dx.doi.org/10.1097/01.aids.0000233579.79714.8d

27. Granich RM, Gilks CF, Dye C, De Cock KM, Williams BG. Universal voluntary HIV testing with immediate antiretroviral therapy as a strategy for elimination of HIV transmission: a mathematical model. Lancet. 2009;373(9657):48-57.
http://dx.doi.org/10.1016/S0140-6736(08)61697-9

28. South African Department of Science and Technology / National Research Foundation Centre for Epidemiological Modelling and Analysis (SACEMA). CEPHIA. Consortium fo the Evaluation and Performance of HIV Incidence Assays. SACEMA. [Accessed 28 June 2012]. Available from: http://www. incidence-estimation.com/page/cephia

29. Le Vu S, Le Strat Y, Barin F, Pillonel J, Cazein F, Bousquet V, et al. F Population-based HIV-1 incidence in France, 2003-08: a modelling analysis. Lancet Infect Dis. 2010;10(10):682-7. http://dx.doi.org/10.1016/S1473-3099(10)70167-5 